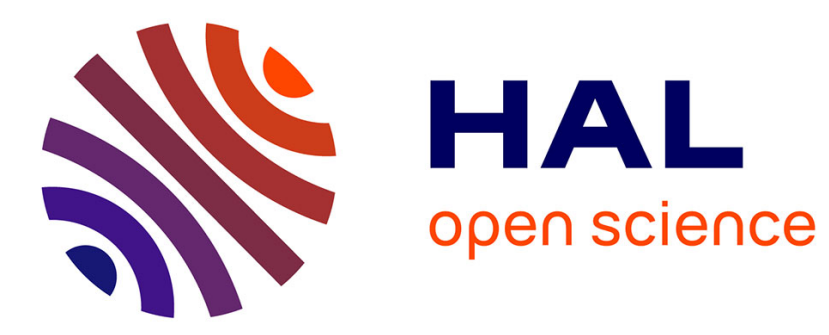

\title{
Dimensional transition in rotating turbulence
}

\author{
E Deusebio, G Boffetta, E Lindborg, S Musacchio
}

\section{To cite this version:}

E Deusebio, G Boffetta, E Lindborg, S Musacchio. Dimensional transition in rotating turbulence. Physical Review E: Statistical, Nonlinear, and Soft Matter Physics, 2014, 90, pp.023005. 10.1103/PhysRevE.90.023005 . hal-01082277

\section{HAL Id: hal-01082277 \\ https://hal.univ-cotedazur.fr/hal-01082277}

Submitted on 13 Nov 2014

HAL is a multi-disciplinary open access archive for the deposit and dissemination of scientific research documents, whether they are published or not. The documents may come from teaching and research institutions in France or abroad, or from public or private research centers.
L'archive ouverte pluridisciplinaire HAL, est destinée au dépôt et à la diffusion de documents scientifiques de niveau recherche, publiés ou non, émanant des établissements d'enseignement et de recherche français ou étrangers, des laboratoires publics ou privés. 


\title{
Dimensional transition in rotating turbulence
}

\author{
E. Deusebio, ${ }^{1,2,{ }^{*}}$ G. Boffetta, ${ }^{3}$ E. Lindborg, ${ }^{2}$ and S. Musacchio ${ }^{4}$ \\ ${ }^{1}$ DAMTP, Centre for Mathematical Sciences, Willbeforce Road, Cambridge CB3 OWA, United Kingdom \\ ${ }^{2}$ Linné Flow Centre, Department of Mechanics, Royal Institute of Technology, 10044 Stockholm, Sweden \\ ${ }^{3}$ Dipartimento di Fisica Generale and INFN, Università di Torino, via Pietro Giuria 1, 10125 Torino, Italy \\ ${ }^{4}$ CNRS, Laboratoire J. A. Dieudonné UMR 6621, Parc Valrose, 06108 Nice, France
}

(Received 20 November 2013; published 13 August 2014)

\begin{abstract}
In this work we investigate, by means of direct numerical hyperviscous simulations, how rotation affects the bidimensionalization of a turbulent flow. We study a thin layer of fluid, forced by a two-dimensional forcing, within the framework of the "split cascade" in which the injected energy flows both to small scales (generating the direct cascade) and to large scale (to form the inverse cascade). It is shown that rotation reinforces the inverse cascade at the expense of the direct one, thus promoting bidimensionalization of the flow. This is achieved by a suppression of the enstrophy production at large scales. Nonetheless, we find that, in the range of rotation rates investigated, increasing the vertical size of the computational domain causes a reduction of the flux of the inverse cascade. Our results suggest that, even in rotating flows, the inverse cascade may eventually disappear when the vertical scale is sufficiently large with respect to the forcing scale. We also study how the split cascade and confinement influence the breaking of symmetry induced by rotation.
\end{abstract}

DOI: 10.1103/PhysRevE.90.023005

PACS number(s): 47.27.ek, 47.27.Gs, 47.32.Ef

\section{INTRODUCTION}

All turbulent processes are characterized by a transfer of kinetic energy among scales. However, depending on the dimension of the space, completely different phenomenology appears. In three dimensions (3D), energy is transferred in a direct cascade towards small scales [1,2], whereas in two dimensions (2D) energy undergoes an inverse cascade towards large scales [3,4]. Numerical simulations have confirmed this picture in both 3D [5] and 2D turbulence [6,7] but they have also shown that the two processes are not mutually exclusive and the coexistence of both a downscale and upscale energy transfer has been observed [8-12].

Previous numerical investigations $[8,10]$ studied the crossover between 2D and 3D dynamics by considering triply periodic domains where one dimension was increasingly contracted. As the ratio $S=L_{z} / L_{f}$ between the geometrical dimension of the compactified direction, $L_{z}$, and the length scale of the forcing, $L_{f}$, decreased below $\sim 0.5$, a mixture of $2 \mathrm{D}$ and $3 \mathrm{D}$ dynamics was observed with a coexistence of a forward and an inverse energy cascade.

In a similar way, also rotation favors a twodimensionalization of the flow, generally supported by the Taylor-Proudman theorem [13]. Experiments of decaying turbulence in rotating tanks show the growth of length scales aligned with the rotation axis, giving evidence that turbulence developing in rotating systems is highly anisotropic. Both linear $[14,15]$ and nonlinear $[16,17]$ mechanisms have been advocated to explain the observed growth. Numerical simulations of rotating flows have also indicated a general trend towards two-dimensionalization [8,17] and some studies [9] have reported a split of the energy cascade into a downscale and an upscale process. Other studies [18] have found that, as the rotation rate $\Omega$ increases, the flows exhibit a dynamics very similar to what is found in a $2 \mathrm{D}$ system, with the vertical

\footnotetext{
*Corresponding author: deusebio@mech.kth.se
}

velocity behaving as a passive scalar [19]. Bourouiba and Bartello [20] reported a strong transfer of energy from 3D modes to 2D modes at intermediate rotation rates, whereas at large rotations a decoupling of the dynamics was observed. The localness of such energy transfer has been investigated in [21].

However, it is not obvious how, starting from a 3D flow fields, the actual two-dimensionalization would occur. The effect of rotation in a turbulent system has recently been a matter of debate. In the strongly rotating limit, energy transfer among scales is supposedly dominated by resonant triad interactions between inertial waves [22]. Even though such interactions can move energy towards the 2D plane [17], triadic resonance cannot transfer energy into/out of the $k_{z}=0$ plane, which, in fact, constitutes a closed resonant set. If only interactions among resonant triads are allowed, 2D modes should thus be dynamically decoupled from 3D modes [18,19]. Sometimes, it is advocated that nearly resonant interactions [17], for which the resonance condition is satisfied only to a certain degree, and higher-order resonance interactions [23] can explain the transfer of energy into the $k_{z}=0$ plane. However, as the flow tends towards two-dimensionalization, the advective time scale, $t_{a} \sim U / l_{h}$, and the inertial wave time scale, $t_{w} \sim l_{z} / \Omega l_{h}$, approach each other and transfer of energy may not only be determined by resonant triads. Cambon et al. [24] suggested that in the framework of wave turbulence theory, two-dimensionalization cannot be achieved for unbounded domains, even in the limit of infinite rotations, and a coupling between 2D and 3D modes always exists.

The interest in 3D dynamics in rotating turbulence has recently been revived also by experiments carried out on a large-scale Coriolis platform. Moisy et al. [25] have shown that flow fields originating from grid-generating decaying turbulence remain highly $3 \mathrm{D}$, even at large rotation rates. Vertical velocity was not behaving as a passive scalar and its coupling with the large-scale flow was suggested to trigger shear instabilities. Thus, it is not clear whether rotating flows would approach a 2D-like dynamics and whether 2D modes would ever dynamically decouple from the 3D flow field. 
The effects of rotations are even more complex in the case in which the flow is confined between two parallel walls perpendicular to the rotation axis. Numerical simulations have shown reflections of inertial waves on the walls and a transition to an almost 2D state [26]. The wave turbulence regime which develops in the limit of strong rotation in a flow confined in the direction of the rotation axis has been recently studied [27].

Another remarkable feature of rotating turbulent flows is the breaking of symmetry between cyclonic and anticyclonic vortices. The predominance of cyclonic vortices (i.e., those which spin in the same direction of $\boldsymbol{\Omega}$ ) has been observed both in experiments [15,25,28-30] and numerical simulations [20,31,32]. Several explanations have been proposed to explain this phenomenon. In particular, it has been shown that the cyclones and anticyclones have different stability properties [31] and different probabilities to be generated at finite Rossby number [33]. Moreover, the correlations between the strain tensor and the vorticity in isotropic turbulence can be responsible for the development of a positive skewness of vertical vorticity when the flow is suddenly subjected to rotation [34]. Interestingly, a similar asymmetry has been observed also in the tropopause [35] and in the stratosphere [36], even though the physical mechanisms acting in the atmosphere are more complex than in idealized rotating turbulence.

Previous works have investigated the dependence of the asymmetry on intensity of rotation. In particular, it has been shown that the skewness of vertical vorticity has a nonmonotonic behavior as a function of the Rossby number [20]. The asymmetry disappears in the limits of both strong and weak rotation and it attains its maximum for intermediate rotation rates [20]. On the contrary, it is still unclear how this asymmetry depends on the aspect ratio $S$ of the flow.

In this paper we investigate how the combined effect of rotation and a periodic confinement affects the turbulent dynamics. We consider the case of intermediate rotation intensities, such that the Coriolis forces are neither too weak to be neglected nor too strong to overwhelm nonlinear interactions. We mainly focus on the transfer of energy and we investigate whether statistically steady regimes, where all the injected energy is transferred towards small scales, can be achieved in rotating confined flows. We also investigate how the parity symmetry breaking on the horizontal plane induced by rotation is affected by the rotation and by the aspect ratio of the flow.

The paper is organized as follows. Section II discusses the numerical code and the parameters used in the simulations. Section III is devoted to the effect of rotation and confinement on the upscale and downscale cascades. Section IV studies the asymmetry in the vorticity field induced by rotation and confinement, and Sec. V is devoted to conclusions.

\section{NUMERICAL SIMULATIONS OF ROTATING TURBULENCE}

We consider the 3D Navier-Stokes equations for an incompressible velocity field $\boldsymbol{u}(\boldsymbol{x}, t)$ (i.e., $\boldsymbol{\nabla} \cdot \boldsymbol{u}=0$ ) in a rotating frame of reference,

$$
\frac{\partial \boldsymbol{u}}{\partial t}+\boldsymbol{u} \cdot \nabla \boldsymbol{u}+2 \boldsymbol{\Omega} \times \boldsymbol{u}=-\nabla p+v \nabla^{2} \boldsymbol{u}+\boldsymbol{f},
$$

where the constant density has been absorbed into the pressure $p, v$ is the kinematic viscosity, and $\boldsymbol{\Omega}=\Omega \boldsymbol{z}$ represents the constant rotation along the vertical direction aligned with the unit vector $z$ [we remark that here "vertical" is used only in analogy with the direction of confinement in experiments as gravity does not explicitly appear in Eq. (1)]. The forcing field $\boldsymbol{f}(\boldsymbol{x}, t)$ is a stochastic Gaussian, white-in-time noise, active only on the horizontal components $u_{x}, u_{y}$ of the velocity and depends on the horizontal components $x, y$ only. The forcing is localized in Fourier space in a narrow band of wave numbers around $k_{f}=2 \pi / L_{f}$ and injects energy into the system at a fixed rate $\varepsilon_{I}[37]$.

Direct numerical simulations (DNSs) of (1) are performed by means of a 2/3-dealiased, parallel, pseudospectral code in a triply periodic domain with various aspect ratios, $r=$ $L_{x} / L_{z}$, and rotations $\Omega$. Simulations are carried out with uniform grid spacing at resolution $N_{x}=N_{y}=r N_{z}$ with fixed $N_{x}=512, L_{x}=2 \pi$, and forcing wave number $k_{f}=8$. The linear rotation and viscous terms are integrated using an exact factor technique, thus removing them from the explicit time integration scheme which uses a second-order Runge-Kutta [38]. The viscous terms in (1) is replaced with a second-order hyperviscosity (Laplacian square) to increase the extension of the inertial range. The hyperviscosity coefficient $v$ is chosen such that $k_{\max } \eta \approx 1.3$, with $\eta=\left(v^{3} / \varepsilon_{I}\right)^{1 / 10}$, similar to what is required in a resolved DNS.

Previous simulations in the absence of rotation $[8,10]$ showed that the ratio between the vertical scale and the forcing scale $S=L_{z} / L_{f}$ controls the relative amount of energy that flows to large scales. In particular, it has been shown that for $S \geqslant 1 / 2$ the inverse energy flux vanishes and the turbulent flow recovers a 3D phenomenology [10]. A second dimensionless parameter in our simulations is provided by the rotation rate which defines a rotation number when made dimensionless with the characteristic time at the forcing scale, $R \equiv \Omega /\left(k_{f}^{2} \varepsilon_{I}\right)^{1 / 3}$ ( $R$ is essentially the inverse of the Rossby number defined as in [17]). In Table I we show the range of parameters in the $(S, R)$ plane in which we performed our simulations.

We study the transition from $2 \mathrm{D}$ to $3 \mathrm{D}$ turbulence by looking at the evolution of the mean kinetic energy $E(t)=$ $\left\langle|\boldsymbol{u}(\boldsymbol{x}, t)|^{2}\right\rangle / 2$ for a flow starting from low-amplitude random noise. For a 3D turbulent flow, after an initial transient, the

TABLE I. Parameter space of the simulations. Each number represents the fraction of energy dissipated at small scales, $\varepsilon_{v} / \varepsilon_{I}$, as a function of $R=\Omega /\left(k_{f}^{2} \varepsilon_{I}\right)^{1 / 3}$ and $S=L_{z} / L_{f}$. The value 1 means that the measured value is compatible with $\varepsilon_{\text {inv }}=0$ and therefore a case with pure 3D phenomenology.

\begin{tabular}{lcccccccccc}
\hline \hline$R \backslash S$ & 0.125 & 0.188 & 0.250 & 0.375 & 0.50 & 0.75 & 1.0 & 2.0 & 4.0 & 8.0 \\
\hline 0.0 & 0.24 & 0.71 & 0.91 & 0.97 & 1 & 1 & 1 & 1 & - & - \\
0.5 & 0.22 & 0.66 & 0.81 & 0.95 & 1 & - & 1 & 1 & - & - \\
0.75 & 0.18 & 0.61 & 0.73 & 0.89 & 0.89 & 0.94 & 1 & 1 & - & - \\
1.0 & 0.17 & 0.45 & 0.64 & 0.76 & 0.77 & 0.82 & 0.86 & 0.87 & 0.95 & 1 \\
1.5 & 0.14 & 0.31 & 0.42 & 0.46 & 0.49 & 0.52 & 0.52 & 0.58 & 0.66 & 0.89 \\
5.0 & - & - & - & - & - & - & 0.02 & 0.02 & 0.08 & 0.14 \\
10.0 & - & - & - & - & - & - & 0.01 & 0.01 & 0.01 & 0.02 \\
\hline \hline
\end{tabular}


mean kinetic energy attains a statistically steady state and the viscous energy dissipation $\varepsilon_{v}=v\left\langle\left(\nabla^{2} \boldsymbol{u}\right)^{2}\right\rangle$ balances the input $\varepsilon_{I}$. In the presence of an inverse cascade, a part of the injected energy, $\varepsilon_{\text {inv }}$, flows to large scales and is not dissipated by viscosity. Energy conservation requires that $\varepsilon_{I}=\varepsilon_{\text {inv }}+\varepsilon_{v}$. Therefore, a signature of an inverse cascade is a linear growth of the mean kinetic energy, $\varepsilon_{\text {inv }}=d E / d t$, and consequently a viscous dissipation $\varepsilon_{v}<\varepsilon_{I}$. We remark that in the presence of an inverse cascade, the flow does not attain a statistically stationary state, as kinetic energy continues to grow and larger and larger scales are generated. This process is nonetheless very slow as the characteristic time grows with the scale $r$ following Kolmogorov scaling $r^{2 / 3}$. Therefore, even in this case we observe a quasistationary regime in which, on the time scale of the largest active modes, small-scale statistics can be averaged.

\section{DIMENSIONAL TRANSITION AND THE EFFECT OF ROTATION}

Figure 1(a) shows the evolution of the mean kinetic energy, $E(t)$, for different values of the aspect ratio $S$ in the absence of rotation $(R=0)$. After an initial transient (of duration independent on $S$ ) in which turbulence develops, we observe a linear growth of the kinetic energy at a rate which is smaller than the input. The linear growth rate, which defines $\varepsilon_{\text {inv }}$, diminishes as the aspect ratio $S$ increases and eventually
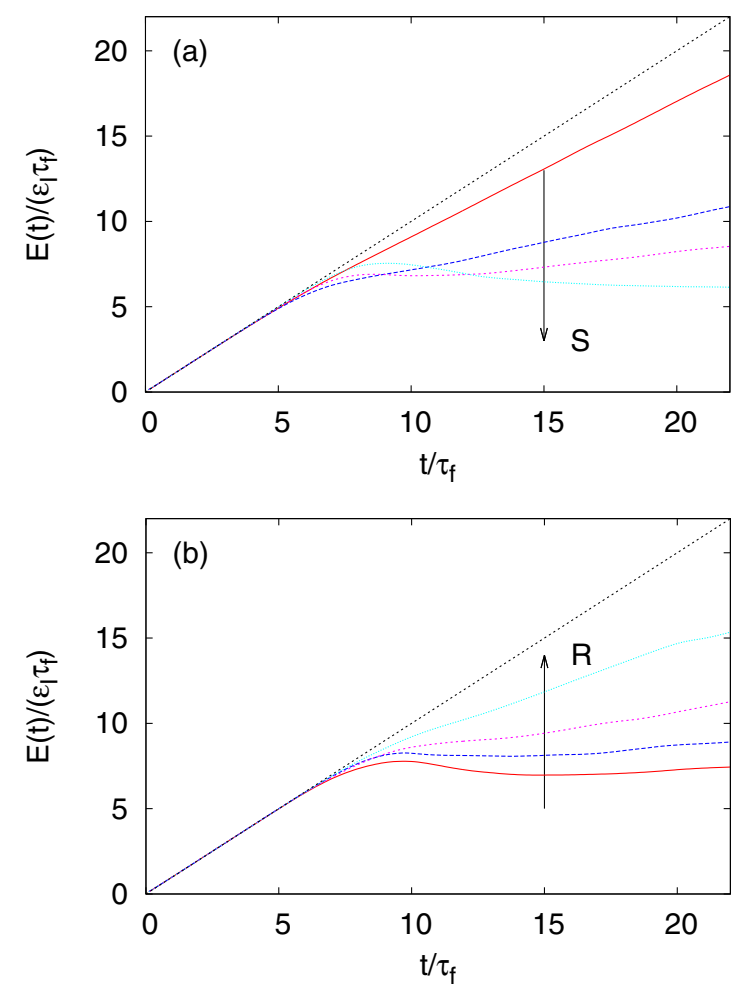

FIG. 1. (Color online) (a) Time evolution of mean kinetic energy for runs at $R=0$ and different values of $S=0.125,0.1875,0.25,0.5$ (from top to bottom). (b) Time evolution of mean kinetic energy for runs at $S=1 / 2$ and different values of $R=0.5,0.75,1.0,1.5$ (from bottom to top). Time is normalized with the characteristic forcing time $\tau_{f}=\left(k^{2} \varepsilon_{I}\right)^{-1 / 3}$. The dotted straight line in both plots represents the energy injection rate.

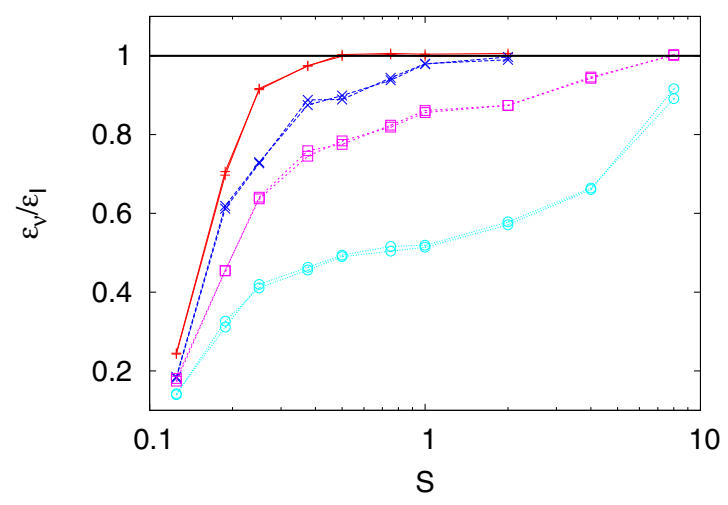

FIG. 2. (Color online) Ratio of small-scale energy dissipation $\varepsilon_{v}$ to the energy input $\varepsilon_{I}$ as a function of the aspect ratio $S$ for different rotation numbers (from top to bottom): $R=0$ (pluses), $R=0.75$ (crosses), $R=1$ (squares), and $R=1.5$ (circles). For each $R$ we plot two values, one given directly by $\varepsilon_{v} / \varepsilon_{I}$, the second by $\varepsilon_{\text {inv }} / \varepsilon_{I}$ in order to have an estimation of the statistical errors.

vanishes for $S \simeq 1 / 2$ as shown in previous work [8,10]. The value of $\varepsilon_{\text {inv }}$ has been estimated with a linear least-squares fit of $E(t)$. We remark that the threshold, $S=1 / 2$, is not expected to be a universal value, as it depends on the particular forcing and also on the precise definition of $k_{f}$. Indeed, it has been shown that different forcing schemes lead to different values of $S$ at which the inverse flux vanishes [8].

Figure 1(b) illustrates the effect of rotation on the split of the energy cascade. At fixed $S=1 / 2$, by increasing $R$ above zero, an inverse cascade is recovered (for $R>0.5$ approximately) with a flux which increases with $R$. This scenario is reproduced at all values of $S$ which we have investigated: For sufficiently large values of rotation an inverse cascade is recovered, as shown in Table I.

We remark that, because we are in quasistationary conditions, we can measure the inverse-cascade flux also as $\varepsilon_{\text {inv }}=$ $\varepsilon_{I}-\varepsilon_{v}$. The two values obtained differ by a few percent, because of the errors in the linear fit of the energy growth and the statistical uncertainty of $\varepsilon_{v}$. In the following we use this difference as a measure of the errors in the calculation of $\varepsilon_{\text {inv }}$ and $\varepsilon_{\nu}$. In Table I the values of $\varepsilon_{v}$ in the $S-R$ parameter space are reported.

The results obtained by this procedure are shown in Fig. 2, where the ratio $\varepsilon_{v} / \varepsilon_{I}$ is plotted as a function of $S$ for different values of $R$. For the case without rotation, $R=0$, we see that for $S \geqslant 0.5$ the inverse cascade vanishes, as shown in Fig. 1(a). Figure 2 shows that an increase of the rotation rate $R$ gives a transition to a pure 3D regime at larger values of $S$. The runs with strong rotation, $R \geqslant 1.5$ (see Table I), show no dimensional transition in $S$. Nonetheless, the small-scale energy dissipation fraction always increases with $S$ and no saturation at a value of $\varepsilon_{v} / \varepsilon_{I}<1$ is evident. This observation suggests that dimensional transition is always present in this system, even for strong rotation rates, for large enough $S$. Of course, this possibility could be confirmed only by increasing $S$ but this would require simulations at larger resolutions (at $S=8$ we already have $N_{z}=N_{x}$ ).

The fact that part of the energy is not dissipated at small scales is not sufficient to guarantee the presence of an inverse 

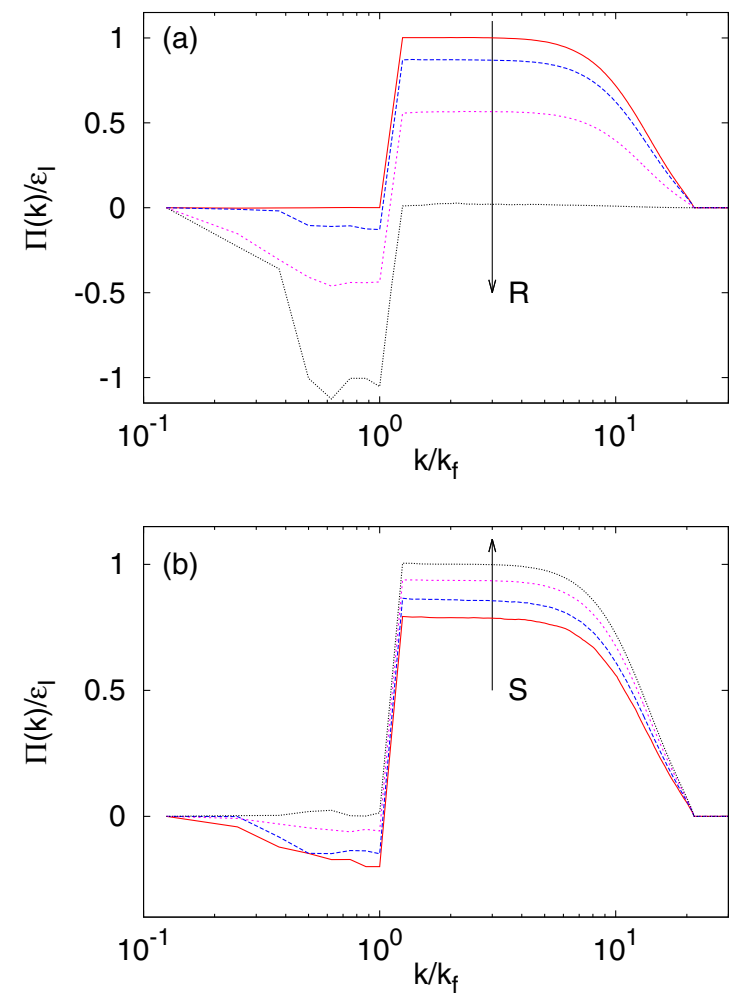

FIG. 3. (Color online) (a) Spectral flux of kinetic energy for different values of $R=0,1,1.5,5$ (following the arrow) at fixed $S=2$. (b) Spectral flux of kinetic energy for different values of aspect ratio $S=0.5,1,4,8$ (following the arrow) at fixed $R=1$. All fluxes have been computed after a steady-state forward cascade has been established and are normalized with energy input $\varepsilon_{I}$.

cascade as observed in 2D turbulence. Indeed, an inverse cascade means a transfer of energy to larger scales which can be observed only by looking at the flux of energy at different wave numbers. We have therefore computed the spectral flux of kinetic energy for different values of $R$ at given aspect ratio $S=2$ [shown in Fig. 3(a)] and for different $S$ at fixed rotation $R=1$ [see Fig. 3(b)]. For $R=0$, because $S=2$ is above the dimensional transition, we have a vanishing inverse flux to wave numbers $k<k_{f}$ and a positive energy fluxes (equal to the energy input) to wave numbers $k>k_{f}$, typical of a 3D scenario with a direct cascade to small scales. By increasing $R$ the flux to small scales reduces (in agreement with Fig. 2); nonetheless, in all cases we observe a clear plateau for wave numbers $k>k_{f}$. It is interesting to observe that for strong rotation rate (run at $S=2$ and $R=5$ ), the direct flux almost vanishes, as predicted in a pure $2 \mathrm{D}$ scenario. At small wave numbers, $k<k_{f}$, and for $R>0$, we observe the development of an inverse cascade produced by rotation. In this range of wave numbers the fluxes are more noisy but nonetheless they are negative, which is the signature of an energy cascade towards large scales. We recall that the range of scales available for the inverse cascade is quite small, since $k_{f}=8$. Figure 3(b) shows the fluxes for different aspect ratios at fixed $R=1$. Again, for all the simulations we have a clear plateau for $k>k_{f}$, at a value $\varepsilon_{v}$ which increases with $S$ (see Fig. 2). The inverse cascade to wave number $k<k_{f}$ is suppressed by increasing $S$ and eventually vanishes for $S=8$.

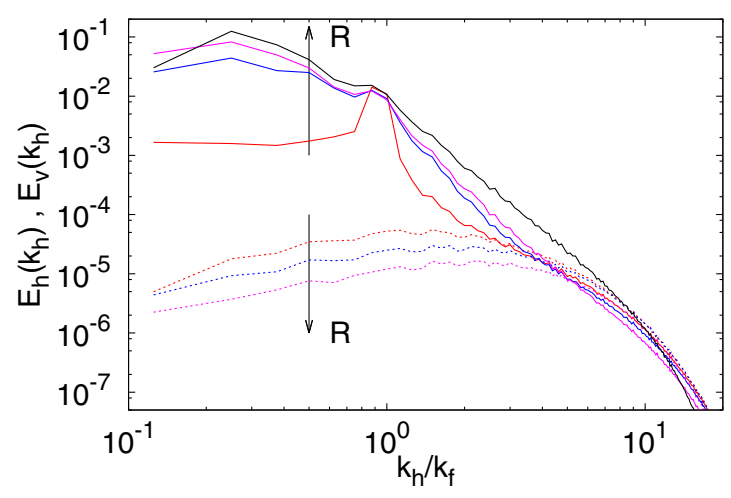

FIG. 4. (Color online) Kinetic energy spectra of the horizontal components $E_{h}\left(k_{h}\right)$ (solid lines) and of the vertical component $E_{v}\left(k_{h}\right)$ (dotted lines) as a function of the horizontal wave number $k_{h}$ with $k_{z}=0$ at $S=2$ for rotations $R=0, R=1.0, R=1.5$, and $R=5$. Rotation increases from bottom to top for continuous lines and decreases from top to bottom for dotted lines, following the arrows (the vertical spectrum at $R=5$ is below $10^{-7}$ and is not shown in the plot). All spectra have been averaged after a steady forward cascade has been established.

Figure 4 shows the kinetic energy spectra corresponding to different simulations at $S=2$. The spectra of both the horizontal components, $E_{h}\left(k_{h}\right)$, and the vertical component, $E_{v}\left(k_{h}\right)$, of the energy are plotted as a function of the horizontal wave number $k_{h}=\sqrt{k_{x}^{2}+k_{y}^{2}}$ with $k_{z}=0$. The horizontal spectra, $E_{h}\left(k_{h}\right)$, display a clear peak around the forcing wave number $k_{f}$ and a narrow power-law scaling at larger wave number. For $k<k_{f}$ the energy spectra $E_{h}\left(k_{h}\right)$ strongly depends on rotation: By increasing $R$ we observe that more energy is present in the large-scale modes. Because of the limited scale separation between the forcing and the box scale $\left(k_{f}=8\right)$ we are not able to observe a clear Kolmogorovlike spectrum in the range of wave numbers $k<k_{f}$.

In the range of small wave numbers, the spectrum of the vertical component, $E_{v}\left(k_{h}\right)$, is strongly suppressed with respect to the horizontal ones, $E_{h}\left(k_{h}\right)$, becoming even smaller as $R$ increases. At large wave number, the vertical spectra become comparable (or even larger) than the horizontal spectra and the $R$ dependence becomes weaker.

The fact that both the confinement and the rotation favor the development of the inverse cascade leads to an interesting consideration. Different flows can have the same ratio between inverse and direct energy fluxes for different values of $S$ and $R$, as evident from Fig. 2. In particular, a nonrotating thin layer can have the same flux ratio as a thick rotating one. In order to understand the similarities and differences between these two cases we need to investigate the mechanisms which are responsible for the transfer of energy towards large scales.

The main difference between 3D and 2D Navier-Stokes equations, written for the vorticity field, is the absence of the vortex stretching term, $\boldsymbol{\omega} \cdot \nabla \boldsymbol{u}$, in the latter. As a consequence, the enstrophy, i.e., mean square vorticity, is conserved in the inviscid limit in 2D flows. In the forced-dissipated case, the presence of two positive-defined inviscid invariants (energy and enstrophy) causes the reversal of the direction of the energy cascade with respect to the $3 \mathrm{D}$ case and the simultaneous 


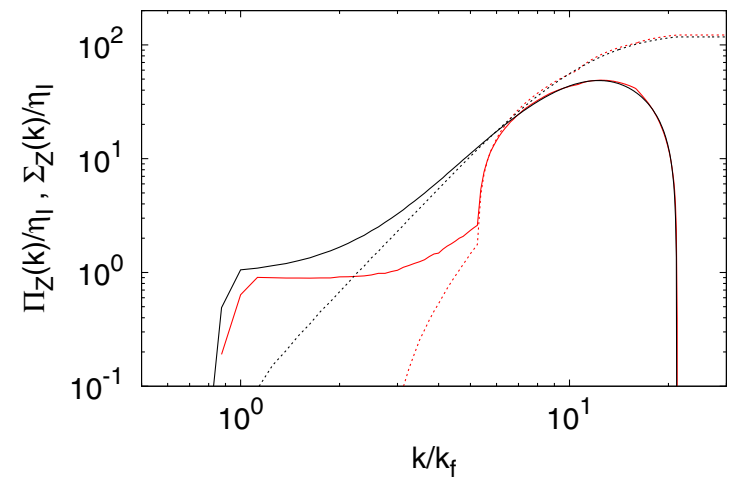

FIG. 5. (Color online) Spectral flux of enstrophy $\Pi_{Z}(k)$ (solid lines) and enstrophy production $\Sigma_{Z}(k)$ (dashed line) for two flow configuration: $R=0, S=0.188$ (lower, red lines) with $k_{z}=42.7$ and $R=1.5, S=4$ (upper, black lines) with $k_{z}=2$. All quantities are normalized with the enstrophy input $\eta_{I}$.

development of a direct enstrophy cascade [3,4]. It is therefore natural to investigate if a similar phenomenology can be observed also in thin fluid layers. In particular, we conjecture that the development of the inverse cascade can be accompanied by a partial suppression of the enstrophy production induced either by the confinement or by the rotation.

To address this issue we computed the spectral flux of enstrophy $\Pi_{Z}(k)$ and enstrophy production $\Sigma_{Z}(k)$ defined as

$$
\begin{aligned}
& \Pi_{Z}(k)=\int_{|\boldsymbol{q}| \leqslant k} \widehat{\boldsymbol{u} \cdot \nabla \boldsymbol{\omega}}(\boldsymbol{q}) \widehat{\boldsymbol{\omega}}^{*}(\boldsymbol{q}) d \boldsymbol{q}, \\
& \Sigma_{Z}(k)=\int_{|\boldsymbol{q}| \leqslant k} \widehat{\boldsymbol{\omega} \cdot \nabla \boldsymbol{u}}(\boldsymbol{q}) \widehat{\boldsymbol{\omega}}^{*}(\boldsymbol{q}) d \boldsymbol{q},
\end{aligned}
$$

where $\widehat{r}$ represents the 3D Fourier transform. In Fig. 5 we show both quantities for a nonrotating thin layer $(R=0, S=$ $0.188)$ and a rotating thick layer $(R=1.5, S=4)$. As shown in Table I, these two flows have approximatively the same ratio between the fluxes of the inverse and direct energy cascade.

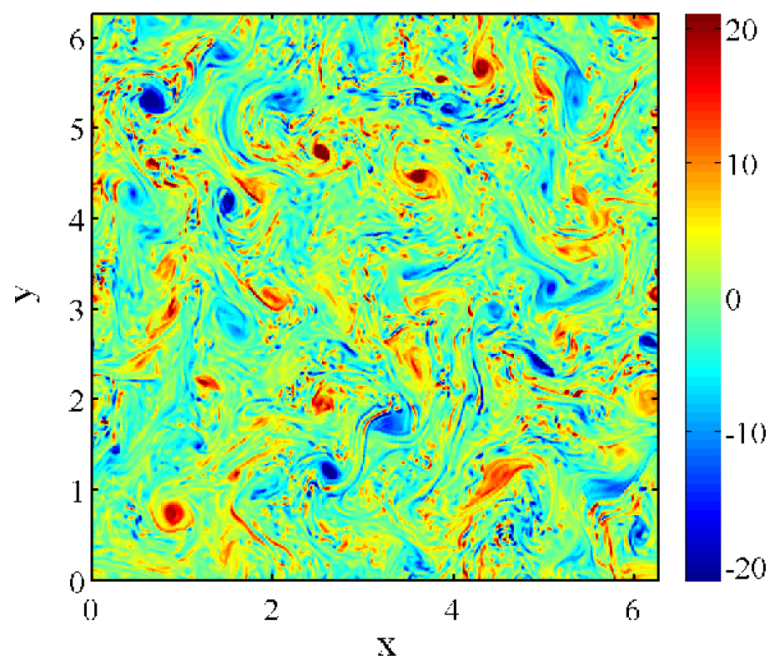

In the nonrotating case we find that the vortex stretching (enstrophy production) is completely suppressed for $k<k_{z} \simeq 5.3 k_{f}\left(k_{z}=2 \pi / L_{z}\right)$ and a direct cascade with almost constant flux of enstrophy is observed for $k_{f}<k<k_{z}$. Therefore, in a thin fluid layer the enstrophy behaves as a quasi-invariant; i.e., it is almost conserved by the dynamics at scales lager than $L_{z}$. In analogy with an ideal 2D flow, the partial conservation of enstrophy is responsible for the development of the inverse energy cascade.

It is worth emphasizing that the total enstrophy behaves as a quasi-invariant, indicating that the development of the inverse energy cascade is not caused directly by the twodimensionalization of the flow, but rather by the presence of a second sign-definite conserved quantity which can be related in spectral space to the energy. Such mechanism is consistent with the previous findings reported in $[39,40]$, which showed that an inverse energy cascade develops also in homogeneous isotropic 3D turbulent flow when mirror symmetry is broken such that helicity has a well-defined sign at all wave numbers.

In the rotating, thick case we observe that the vortex stretching is also suppressed, but this phenomenon occurs on a broad range of scales and there is no evidence of an inertial range in which enstrophy is conserved. Our findings seem to indicate that, unlike confinement, moderate rotation is not sufficient to develop a direct cascade of enstrophy with constant flux. Nevertheless, the mechanism by which rotation enhances the transfer of energy towards large scales is similar to the one which is induced by confinement, i.e., via suppression of the vortex stretching term. It is worth noticing that the enstrophy production and enstrophy flux of the two cases are almost indistinguishable at small scales.

\section{CYCLONIC-ANTICYCLONIC ASYMMETRY}

The breaking of asymmetry between cyclonic and anticyclonic vortices, which has been observed both in experiments [15,25,28-30] and in numerical simulations [20,31,32], is a distinctive feature of rotating turbulent flows. Here we are interested to investigate how this asymmetry is influenced both by the rotation and the confinement of the flow. In Fig. 6

FIG. 6. (Color online) Horizontal cuts of vertical vorticity $\omega_{z}$ for two flow configurations $(R=0, S=0.188)($ left $)$ and $(R=1.5, S=4)$ (right).

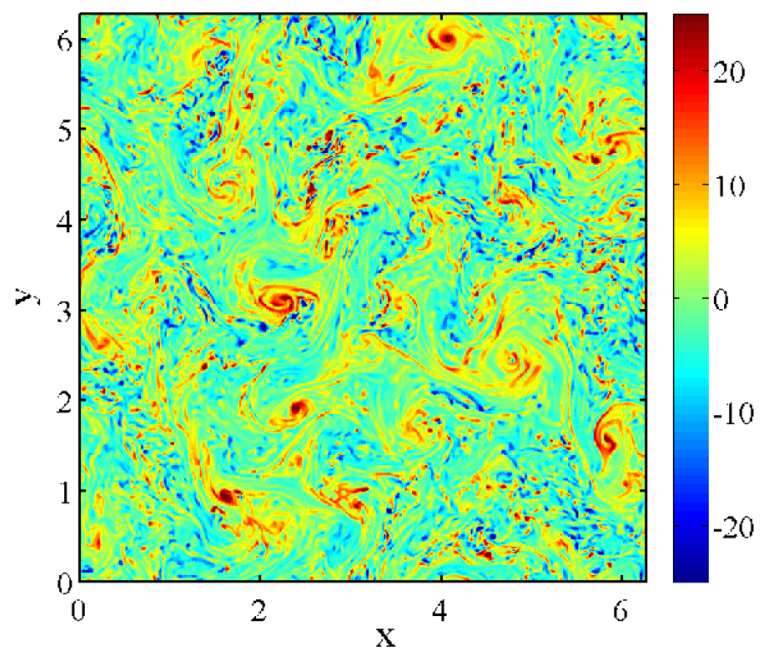




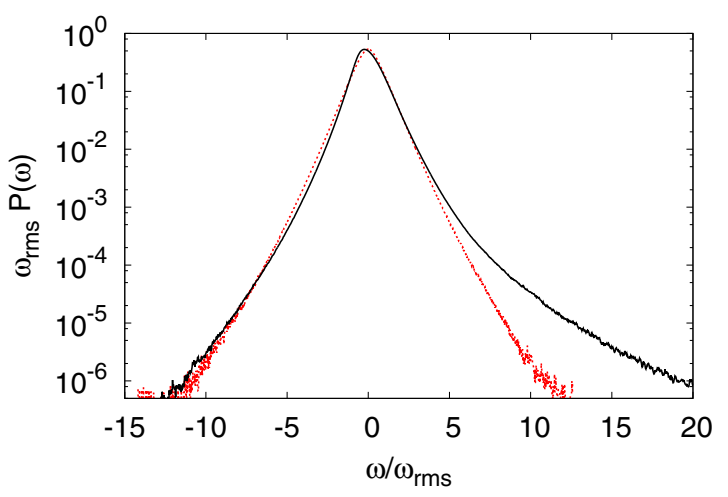

FIG. 7. (Color online) PDFs of vertical vorticity for $S=2$, $R=0$ (red dotted line) and $S=2, R=1.5$ (black solid line).

we show two horizontal cuts of the vertical component of the vorticity, $\omega_{z}$, for the two runs discussed in Sec. III, i.e., a thin nonrotating layer and a thick rotating layer with a similar fraction of energy dissipated at small scales. In the nonrotating, thin layer $(R=0, S=0.188$, left panel) vortices with positive and negative sign are equally distributed. In contrast to this, the thick rotating flow $(R=1.5, S=4$, right panel) shows a clear asymmetry between cyclonic and anticyclonic vortices. In particular, the cyclonic vortices are enhanced while anticyclonic vortices are suppressed.

In Fig. 7 we compare the probability density function (PDF) of vertical vorticity of the two flows $P(\omega)$. In the nonrotating case the PDF is symmetric, while in the rotating case it shows a clear asymmetry. Cyclonic vortices, corresponding to the right tail, are much more probable than anticyclonic ones. We remark that in both cases the mean vertical vorticity $\omega_{z}$ vanishes and therefore a quantitative measure of the asymmetry is provided by the skewness,

$$
S_{\omega}=\frac{\left\langle\omega_{z}^{3}\right\rangle}{\left\langle\omega_{z}^{2}\right\rangle^{3 / 2}} .
$$

In the nonrotating case $S_{\omega}=0$ while in the rotating cases one has $S_{\omega}>0$. Figure 7 suggests that a significant contribution to the skewness $S_{\omega}$ comes from the tails of the PDF of $\omega_{z}$. The $R$ dependence of the skewness is shown in Fig. 8(a) and it is found to be nonmonotonic, with an increase for moderate rotations followed by a subsequent decrease for larger rotation rates. This is in agreement with previous findings [20] for which the strongest symmetry breaking is observed at intermediate $R$, corresponding to $R o=0.2$.

It is interesting to point out that the asymmetry also depends on the aspect ratio $S$ of the flow. Indeed, in the 2D limit $(S \rightarrow 0)$ rotation cannot induce an asymmetry in $\omega_{z}$ as the $\boldsymbol{\Omega} \times \mathbf{u}$ term disappears in the $2 \mathrm{D}$ version of the Navier-Stokes equation (1). Therefore, we expect the skewness $S_{\omega}$ to be an increasing function of $S$ at fixed $R$. This is confirmed by our numerical results, as shown in Fig. 8(b). Further, our findings suggest that for $S>1$ the skewness saturates as $S$ increases, although we cannot exclude a different behavior for values of $S$ larger than 8 . That the asymmetry vanishes for $S \rightarrow 0$ also suggests a possible interpretation of the decreases of the skewness for large $R$ as a signature of the bidimensionalization of the flow.
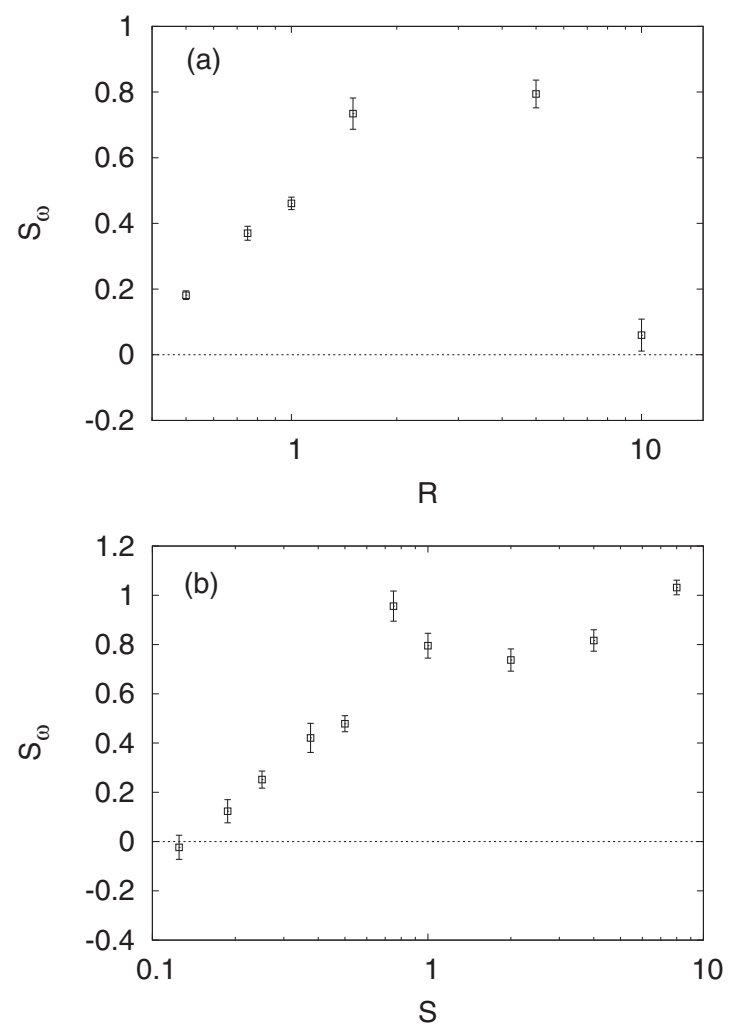

FIG. 8. Skewness of vertical vorticity $S_{\omega}$ as a function of (a) rotation $R$ for fixed $S=2$ and (b) $S$ at fixed $R=1.5$. The error bars represent the error on the mean value over a time interval.

The asymmetry in the cyclonic-anticyclonic motions does not manifests only at small scales but also on large-scale structures, as evident in Fig. 6. In 3D turbulence, the leading contributions to the vorticity field come from small-scale structures, and therefore a criterion based on (4) could be not adequate to capture the asymmetry at large scale. Moreover, it is likely to be influenced by the Reynolds number as $\omega_{z}$ is a small-scale quantity. An alternative measure of the cyclonicanticyclonic asymmetry can be provided by the skewness of the azimuthal velocity increment $\delta u_{T}$,

$$
S_{T}=\frac{\left\langle\delta u_{T}^{3}\right\rangle}{\left\langle\delta u_{T}^{2}\right\rangle^{3 / 2}},
$$

where $\delta u_{T}=[\boldsymbol{u}(\boldsymbol{x}+\boldsymbol{r})-\boldsymbol{u}(\boldsymbol{x})] \cdot \boldsymbol{t}$, with $\boldsymbol{t}$ being the horizontal unit vector in the cyclonic direction; that is, $(\boldsymbol{r} / r, \boldsymbol{t}, \boldsymbol{z})$ forms a orthogonal right-handed system of reference. As opposed to (4), (5) is a scale-dependent quantity and it is thus more informative. The statistics of azimuthal velocity increment has been fruitfully used to analyze measurements in the stratosphere [41] and numerical simulations of rotating and stratified turbulence [42], which show quadratic dependence in $r$.

In our simulations, we find positive values of $S_{T}$ of the order of unity for rotating flows (Fig. 9), indicating that cyclonic motions are dominating at all scales. As for the skewness of vorticity, also in this case we observe a nonmonotonic behavior of $S_{T}$ with $R$ [the maximum value is reached for $R=O(1)]$. Moreover, for all the values $R>0$, we observe 


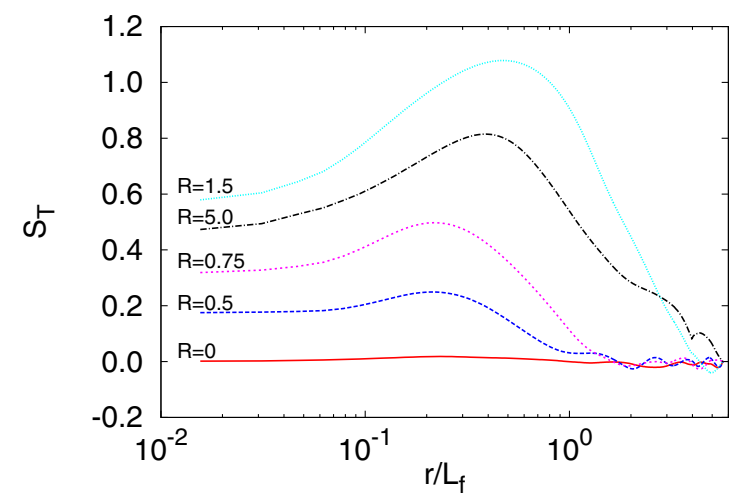

FIG. 9. (Color online) Skewness of the transversal velocity increments $S_{T}=\left\langle\delta u_{T}^{3}\right\rangle /\left\langle\delta u_{T}^{2}\right\rangle^{3 / 2}$ for $S=2$ and $R=0,0.5,0.75,1.5,5$.

a $r$ dependence of the skewness which shows a maximum at a scale which increases with $R$. It is worth noticing that for $R>1$ the asymmetry persists at scales larger than the forcing scale $L_{f}$, i.e., in the energy inverse-cascade range.

\section{CONCLUSION AND DISCUSSION}

In this work we investigated by means of numerical simulations how rotation affects the turbulent cascade of kinetic energy in a thin fluid layer. We have shown that rotation enhances the inverse energy cascade. This is achieved by a mechanism which is similar to what is observed when the flow is confined, i.e., by suppressing the production of enstrophy at large scales. For thick fluid layers such suppression is sufficient to allow the development of an inverse cascade even in cases in which it would not be observable in the absence of rotation. On the other hand, for a fixed rotation number, we observe that increasing the aspect ratio causes a suppression of the inverse energy cascade.

Rotation also breaks the symmetry of horizontal flow, inducing a predominance of cyclonic vortices over anticyclonic ones. Our results confirm that this asymmetry is maximum at intermediate rotation rates and vanishes for both weak and strong rotation. The analysis of the skewness of transverse velocity structure functions reveals that the asymmetry is not only present at small scales, but can be observed also in large-scale structures of the flow. Interestingly, we find that the cyclonic-anticyclonic asymmetry at fixed rotation vanishes as the thickness of the fluid layer is reduced, consistent with the fact that in ideal 2D flows the rotation effects disappear.

When carrying out numerical simulations and experiments in rotation fluids, great care should thus be taken in setting up the boundary conditions in the direction parallel to the rotation axis. In experiments, bottom and top Ekman boundary layers can strongly influence the dynamics [29,43,44]. Numerical simulations can similarly be affected by the finite size of computational domains and confinement effects can effectively influence the dynamical picture. In rotating flows, bounded domains may also set the minimum frequency for nonstationary inertial waves, $\omega \sim \Omega k_{z, \min } / k$. This value can nevertheless be very large at large rotation rate, therefore enforcing a decoupling with the $2 \mathrm{D}$ modes which have zero frequency.

Our findings pose interesting questions. In particular, one may ask whether there would always exist, irrespective on how large the rotation rate is, a dimensional transition between a $2 \mathrm{D}$ dynamics, with an inverse energy cascade, and a 3D dynamics, with a direct energy cascade. More generally, it would be interesting to derive theoretical predictions on the scaling of the critical curve in the $(S, R)$ parameter space which borders the region in which the inverse cascade exists.

\section{ACKNOWLEDGMENTS}

Computer time provided by SNIC (Swedish National Infrastructure for Computing) and by Cineca is gratefully acknowledged. S. Musacchio wishes to thank F. S. Godeferd for fruitful discussions. The authors thank an anonymous reviewer for valuable comments and suggestions.
[1] A. Monin and A. Yaglom, Statistical Fluid Mechanics (MIT Press, Cambridge, MA, 1971).

[2] U. Frisch, Turbulence: The Legacy of AN Kolmogorov (Cambridge University Press, Cambridge, UK, 1995).

[3] R. Kraichnan, Phys. Fluids 10, 1417 (1967).

[4] G. Boffetta and R. Ecke, Annu. Rev. Fluid Mech. 44, 427 (2012).

[5] T. Ishihara, T. Gotoh, and Y. Kaneda, Annu. Rev. Fluid Mech. 41, 165 (2009).

[6] G. Boffetta and S. Musacchio, Phys. Rev. E 82, 016307 (2010).

[7] A. Vallgren and E. Lindborg, J. Fluid Mech. 671, 168 (2011).

[8] L. M. Smith, J. R. Chasnov, and F. Waleffe, Phys. Rev. Lett. 77, 2467 (1996).

[9] P. D. Mininni and A. Pouquet, Phys. Rev. E 79, 026304 (2009).

[10] A. Celani, S. Musacchio, and D. Vincenzi, Phys. Rev. Lett. 104, 184506 (2010).

[11] A. Vallgren, E. Deusebio, and E. Lindborg, Phys. Rev. Lett. 107, 268501 (2011).
[12] E. Deusebio, A. Vallgren, and E. Lindborg, J. Fluid Mech. 720, 66 (2013).

[13] G. I. Taylor, Proc. R. Soc. London, Ser. A 104, 213 (1923).

[14] P. Davidson, P. Staplehurst, and S. Dalziel, J. Fluid Mech. 557, 135 (2006).

[15] P. Staplehurst, P. Davidson, and S. Dalziel, J. Fluid Mech. 598, 81 (2008).

[16] C. Cambon, N. Mansour, and F. Godeferd, J. Fluid Mech. 337, 303 (1997).

[17] L. Smith and F. Waleffe, Phys. Fluids 11, 1608 (1999).

[18] Q. Chen, S. Chen, G. Eyink, and D. Holm, J. Fluid Mech. 542, 139 (2005).

[19] P. Embid and A. Majda, Geophys. Astrophys. Fluid Dyn. 87, 1 (1998).

[20] L. Bourouiba and P. Bartello, J. Fluid Mech. 587, 139 (2007).

[21] L. Bourouiba, D. N. Straub, and M. L. Waite, J. Fluid Mech. 690, 129 (2012).

[22] F. Waleffe, Phys. Fluids 5, 677 (1993). 
[23] A. C. Newell, J. Fluid Mech. 35, 255 (1969).

[24] C. Cambon, R. Rubinstein, and F. S. Godeferd, New J. Phys. 6, 73 (2004).

[25] F. Moisy, C. Morize, M. Rabaud, and J. Sommeria, J. Fluid Mech. 666, 5 (2011).

[26] F. S. Godeferd and L. Lollini, J. Fluid Mech. 393, 257 (1999).

[27] J. F. Scott, J. Fluid Mech. 741, 316 (2014).

[28] E. J. Hopfinger, F. K. Browand, and Y. Gagne, J. Fluid Mech. 125, 505 (1982).

[29] E. J. Hopfinger and G. J. F. van Heijst, Annu. Rev. Fluid Mech. 25, 241 (1993).

[30] C. Morize, F. Moisy, and M. Rabaud, Phys. Fluids 17, 095105 (2005).

[31] P. Bartello, O. Metais, and M. Lesieur, J. Fluid Mech. 273, 1 (1994).

[32] L. J. A. van Bokhoven, C. Cambon, L. Liechtenstein, F. S. Godeferd, and H. J. H. Clercx, J. Turbul. 9, N6 (2008).

[33] B. Sreenivasan and P. A. Davidson, Phys. Fluids 20, 085104 (2008).
[34] J.-N. Gence and C. Frick, C. R. Acad. Sci., Ser. IIb: Mec. 329, 351 (2001).

[35] G. J. Hakim and A. K. Canavan, J. Atmos. Sci. 62, 231 (2005).

[36] E. Lindborg and J. Y. N. Cho, J. Geophys. Res.: Atmos. 106, 10233 (2001).

[37] E. Lindborg and G. Brethouwer, J. Fluid Mech. 586, 83 (2007).

[38] C. Canuto, M. Y. Hussaini, A. Quarteroni, and T. A. Zang, in Spectral Methods in Fluid Dynamics (Springer-Verlag, Berlin, 1988), pp. 1-567.

[39] L. Biferale, S. Musacchio, and F. Toschi, Phys. Rev. Lett. 108, 164501 (2012).

[40] L. Biferale, S. Musacchio, and F. Toschi, J. Fluid Mech. 730, 309 (2013).

[41] J. Y. N. Cho and E. Lindborg, J. Geophys. Res. 106, 10223 (2001).

[42] E. Deusebio, P. Augier, and E. Lindborg, J. Fluid Mech. (2014), doi:10.1017/jfm.2014.414.

[43] A. Ibbetson and D. J. Tritton, J. Fluid Mech. 68, 639 (1975).

[44] C. Morize and F. Moisy, Phys. Fluids 18, 065107 (2006). 